\title{
Evaluation and preservation of fertility in adolescent and young adult patients with testicular cancer
}

This article was published in the following Dove Press journal:

Clinical Oncology in Adolescents and Young Adults

19 March 2013

Number of times this article has been viewed

\author{
Jennifer M Levine' \\ Alison E Fernbach' \\ Peter J Stahl ${ }^{2}$ \\ 'Department of Pediatrics, \\ ${ }^{2}$ Department of Urology, Columbia \\ University Medical Center, New York, \\ NY, USA
}

\begin{abstract}
Testicular cancer is the most common cancer affecting young adult males. With survival rates in the range of $70 \%-99 \%$ depending on stage, it is critical to address the long-term consequences of diagnosis and treatment. Maintaining the ability to have biologic children has been identified as an area of importance for long-term survivors of testicular cancer. Yet many males will face a risk of infertility as a consequence of the surgical, radiotherapeutic, and chemotherapeutic treatments used to cure them of their cancer. Attention to fertility preservation in the form of sperm banking prior to the start of therapy in those individuals able to produce sperm is a vital intervention in this population. For those individuals who are not able to produce sperm, knowledge and access to alternative methods of fertility preservation and parenthood is necessary to provide comprehensive care. This review summarizes the issues regarding fertility evaluation and preservation in adolescents and young adults with testicular cancer.
\end{abstract}

Keywords: fertility, adolescent and young adult, testicular cancer

\section{Introduction}

In the United States, more than 8000 males will develop testicular cancer annually, making it the most common cancer diagnosis in the young adult male population. ${ }^{1}$ Unlike cancers that mainly affect pediatric patients or older adults, individuals diagnosed with testicular cancer are most commonly between the ages of 20 and 39 years, a time when reproduction is a major life focus. ${ }^{2}$ With overall survival rates approaching $96 \%$ and the possibility that disease status and curative treatments may be toxic to reproductive function, it is not surprising that fertility and parenthood are important issues to survivors of cancer, ${ }^{3}$ and that for some the experience of having had cancer increases the value they place on parent-child relationships. ${ }^{4}$ Understanding the impact that cancer-directed therapy can have on gonadal function and the methods that exist to preserve fertility is necessary to maximize an individual testicular cancer survivor's chance of having biologic children in the future. ${ }^{5}$

\section{Normal sperm development}

Spermatogenesis, the maturation of haploid spermatozoa from immature spermatogonial germ cells, occurs in the intraluminal-compartment seminiferous tubules of the testes with the support of Sertoli cells. This process depends upon high intratesticular levels of testosterone, which promote sperm production and are essential for the development of secondary sex characteristics. Testosterone is produced by interstitial Leydig cells that surround the epithelium. At the start of puberty, the hypothalamus
Correspondence: Jennifer Levine 16I Fort Washington Avenue, IP-7, New York, NY 10032, USA

$\mathrm{Tel}+\mathrm{I} 2 \mathrm{I} 23052368$

Fax +I 2123055848

Email jl175@columbia.edu 
produces gonadotropin-releasing hormone, which causes the anterior pituitary to release follicle-stimulating hormone (FSH) and luteinizing hormone. FSH primarily influences the function of the Sertoli cells, while luteinizing hormone drives the Leydig cells. With the onset of this hormonal activity, the formerly dormant germ cells begin the subsequently lifelong process of self-renewal and differentiation into spermatogonia. ${ }^{6,7}$ The full process of maturation to haploid spermatozoa takes approximately 74 days. Spermatozoa are stored in the epididymis until ejaculation (Figure 1).

\section{Risks for infertility}

It is not uncommon for reduced spermatogenesis to be already present at the time of diagnosis of testicular cancer. ${ }^{8}$ Although the etiology and correlation are not well defined, individuals who are infertile have a significantly higher risk of developing testicular cancer compared to fertile men. ${ }^{9,10}$ Treatment for testicular cancer can cause or exacerbate diminished fertility. While most men will become azoospermic due to interruptions in spermatogenesis during treatment, the risk for permanent infertility is related to the degree of depletion of the relatively quiescent spermatogonial stem cells and to disruption of normal anatomic and nerve function. Although reasons for interindividual variations have not been well elucidated, there appear to be host factors related to infertility risk, as not all individuals exposed to the same gonadotoxic drugs and doses will have the same response. Overall rates of conception in testicular cancer survivors attempting to conceive have been reported to be between $50 \%$ and $100 \%$, varying widely depending on treatment, with time from diagnosis to conception of a first child of approximately 7-12 years. ${ }^{11-13}$

\section{Surgery}

When used as the sole treatment modality, unilateral orchiectomy should preserve the capacity to sire a child. In the instances where bilateral orchiectomies are necessary, there are obvious implications for future fertility. Bilateral retroperitoneal lymph-node dissection (RPLND) without nerve sparing can result in retrograde ejaculation and

Spermatogenesis

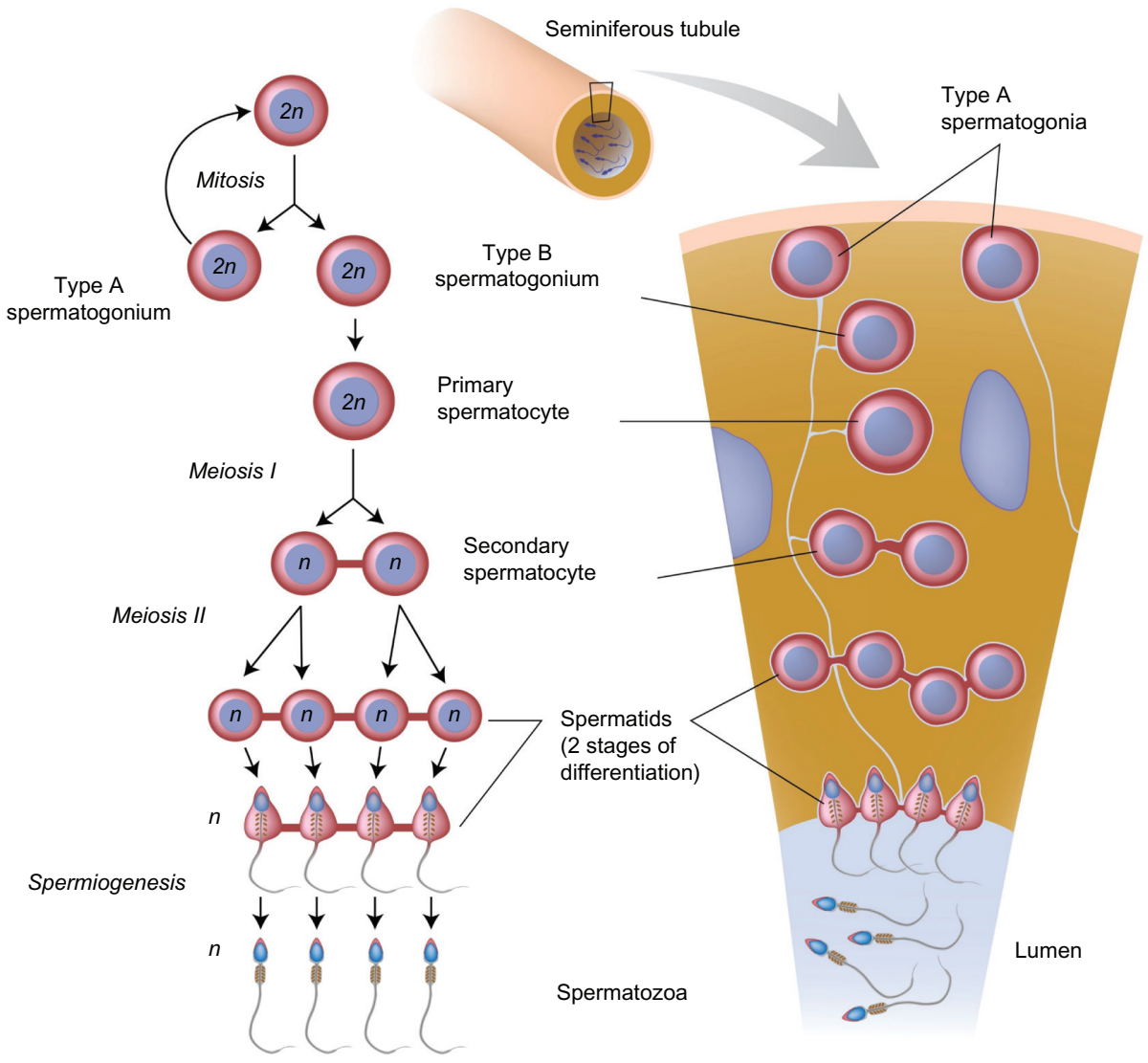

Figure I Spermatogenesis.

(c) Nguyet M Le/I 23FR.com. 
subsequent impaired fertility in greater than $90 \%$ of patients. The use of techniques that spare the nerves reduces this risk considerably. ${ }^{14}$

\section{Chemotherapy}

Most chemotherapy agents are toxic to maturing sperm, resulting in impaired spermatogenesis and at least temporary azoospermia during and immediately after treatment. The length of time that oligospermia or azoospermia persists (including becoming permanent) appears to be related to total cumulative doses of chemotherapy received and likely reflects the degree to which the quiescent germ cells have been affected. Alkylating agents, such as cyclophosphamide, ifosfamide, and busulfan are the agents most likely to result in permanent infertility. Cumulative doses of cyclophosphamide exceeding $7.5 \mathrm{~g} / \mathrm{m}^{2}$ are considered the threshold for infertility, ${ }^{15}$ although there is some evidence that even at much higher doses of cyclophosphamide, fertility can resume over time. ${ }^{6}$ Cisplatin, an agent commonly used in the treatment of testicular cancer, has also been associated with significant gonadal toxicity usually at cumulative doses exceeding $600 \mathrm{mg} / \mathrm{m}^{2}$, with increased risk for toxicity associated with higher cumulative doses. A study of paternity and testicular function in testicular cancer survivors with a median follow-up of 12 years who had received two $(n=2)$, three $(n=30)$, or four $(n=68)$ cycles of chemotherapy containing cisplatin demonstrated paternity rates of $100 \%$, $83 \%$, and $73 \%$, respectively, the vast majority without using cryopreserved sperm. ${ }^{16}$ However, in a study evaluating paternity in those treated with multimodality therapy that included higher-dose cisplatin $(>850 \mathrm{mg})$, the paternity rate was $48 \% .^{17}$

In general, germ cells are more sensitive to the effects of chemotherapy than Leydig cells, making azoospermia a more frequent long-term complication than testosterone deficiency. ${ }^{6}$ When testosterone production is impaired, however, males may require testosterone supplementation to maintain libido, erectile function, bone density, and muscle mass. In those males who have not yet gone through puberty and have Leydig cell damage, supplementation may be required for the development of secondary sex characteristics. ${ }^{18}$

\section{Radiation}

Testicular function is highly affected by even small doses of radiation, such that either direct irradiation or irradiation from scatter can lead to infertility. Like chemotherapy exposure, the degree of toxicity and time to recovery is dose-dependent, with higher doses leading to permanent azoospermia.
Increasing fractionation also appears to increase damage. ${ }^{19}$ Doses as low as $0.1 \mathrm{~Gy}$ to the seminiferous tubules can cause temporary azoospermia in the order of 6 months, doses in the 2-3 Gy range can have a longer-term impact with recovery seen around 30 months, while doses in the range of 6 Gy and greater can lead to permanent infertility by causing significant depletion of the germ cell pool. ${ }^{19,20}$ As with chemotherapy, Leydig cells are susceptible to damage from irradiation but less so than stem cells, with doses of 20-30 Gy leading to dysfunction. ${ }^{21}$

\section{Fertility evaluation}

Evaluation of fertility in adolescents and young adult patients with testicular cancer provides important clinical information that guides selection of fertility-preservation strategies. Fortunately, in most cases semen analysis performed concurrently with attempted cryopreservation of ejaculated sperm ("sperm banking") indicates the presence of sufficient motile sperm to obviate the need for a more extensive fertility evaluation. In some cases, however, the patient may be anejaculatory and unable to provide a semen sample. In other cases, semen analysis may indicate an insufficient quantity of motile sperm to meet the patient's future reproductive goals. The latter scenario is common in testicular cancer patients, in whom the estimated prevalence of oligospermia (low sperm concentration) and azoospermia (zero detectable sperm in the ejaculate) are $50 \%$ and $10 \%-15 \%$, respectively, at diagnosis. ${ }^{22,23}$ In such cases, the history, physical examination, and hormonal evaluation are informative about the prognosis and optimal method for sperm acquisition.

\section{Semen analysis}

Semen analysis is the first step in the fertility evaluation of candidates for fertility preservation. Interpretation is performed according to reference limits published by the World Health Organization (WHO), most recently in $2010 .{ }^{24}$ Semen characteristics reported include semen volume (normal $\geq 1.5 \mathrm{~mL}$ ), sperm concentration (normal $\geq 15$ million sperm $/ \mathrm{mL}$ ), total sperm number (normal $\geq 39$ million), \% of sperm with overall motility (normal $\geq 40$ ), $\%$ of sperm with forwardprogressive motility (normal $\geq 32 \%$ ), and $\%$ of sperm with strictly normal morphological features (normal $\geq 4 \%$ ).

The adequacy of a semen sample for fertility preservation must be determined on an individualized, case-by-case basis that takes each patient's reproductive goals into consideration. In general, the goal is cryostorage of several million viable sperm divided into as many separate aliquots as possible, 
as each attempt at assisted reproduction generally requires thawing one aliquot of sperm. Motility may reasonably be used as a surrogate measure of viability, as motile sperm are alive by definition. Specific sperm-viability testing is also available and is particularly useful when the percentage of motile sperm is low. All semen samples containing any viable sperm should be cryopreserved, because fertilization and pregnancy rates using contemporary assisted reproductive techniques such as intracytoplasmic sperm injection (ICSI) are largely unaffected by semen quality. ${ }^{25}$

It is, however, particularly important to identify fertilitypreservation candidates that may not have access to advanced assisted reproductive technologies. In these patients, the only options for future reproduction are intravaginal or intrauterine insemination (IUI) of thawed, cryopreserved sperm. Whereas ICSI requires only one sperm per available oocyte for fertilization and reproduction, IVI and IUI typically require at least 500,000 motile sperm per attempt. Therefore, a single semen sample containing 1 million sperm with $30 \%$ motility is more than adequate for fertility preservation in a patient with future access to ICSI, but may not be adequate in the patient that does not have access to advanced assisted reproductive methods.

\section{History}

The history of an adolescent or young adult who is a candidate for sperm-acquisition procedures should focus upon the medical, surgical, and developmental histories. ${ }^{26}$ Medical comorbidities and their treatments (ie, prior chemotherapy or irradiation) may negatively impact fertility and must be identified. Patients should be directly questioned about pediatric inguinal and scrotal operations, including hydrocelectomy, herniorrhaphy, and orchidopexy (common in testicular cancer patients given the three to eightfold increased risk of germ cell cancer associated with cryptorchidism ${ }^{27}$ ). These procedures may cause iatrogenic obstructive azoospermia, due to injury or ligation of the epididymis or vas deferens. ${ }^{28}$ It is also important to identify patients who have been treated with RPLND for retroperitoneal lymphatic metastases. RPLND may cause surgical disruption of the sympathetic chains or postganglionic branches of the lumbar sympathetic nerves, and is a common cause of retrograde ejaculation and anejaculation. Lastly, the developmental history is particularly important when evaluating peripubertal adolescents. The patient should be questioned about their familiarity with masturbation, and whether or not they have ever ejaculated during sexual activity or experienced nocturnal seminal emission. This discussion should occur privately with the adolescent patient after obtaining appropriate parental consent.

\section{Physical examination}

The most important components of the physical examination include assessment of the genital developmental stage (Tanner genital stage), assessment of testicular consistency and volume, and palpation of the vasa deferentia and epididymities. Sperm acquisition is not indicated in patients in whom pubertal development has not begun, but may be successful even in early puberty. ${ }^{29}$ It is therefore useful to broadly characterize peripubertal adolescents as either prepubertal (Tanner genital stage 1) or pubertal (Tanner genital stage 2 or greater).

Testicular volume is best assessed with a Prader orchidometer and is a key indicator of sperm production, as the majority of testicular volume is comprised of the spermproducing seminiferous tubules. Postpubertal adolescents and adults with small, soft testicles usually have impaired spermatogenesis. In contrast, the presence of normal testicular size and consistency suggests a normal volume of seminiferous tubular tissue and raises the possibility of obstruction of the male genital tract in an individual with impaired fertility. In peripubertal adolescents, testicular volume is also an indicator of pubertal status. Successful sperm acquisition has been described in adolescents who have entered puberty and have testicular volumes greater than $5 \mathrm{~mL} /$ testis, $^{29}$ irrespective of chronological age.

Each vas deferens should be easily palpated within the spermatic cord through the scrotal skin. Congenital vasal agenesis is a relatively common cause of obstructive azoospermia that is diagnosed on physical examination. This finding usually reflects a mild form of cystic fibrosis and should prompt genetic testing for abnormalities in the cystic fibrosis transmembrane conductance receptor, which may be relevant to future offspring conceived with surgically retrieved sperm. Lastly, the epididymities are palpated for fullness and induration that if present are highly suggestive of obstructive azoospermia due to vasal or ejaculatory duct obstruction.

\section{Endocrine evaluation}

The diagnostic evaluation of azoospermic, severely oligozoospermic, or anejaculatory testicular cancer patients considering sperm-acquisition procedures should include measurement of the serum testosterone (T) and FSH. These tests are most useful in azoospermic postpubertal adolescents and adults, in whom they may be interpreted in combination 
with the history and physical examination to distinguish poor sperm production from obstruction. The most common finding in azoospermic testicular cancer patients is primary testicular failure $(\mathrm{FSH}>8 \mathrm{IU} / \mathrm{L} \pm \mathrm{T}<300 \mathrm{ng} / \mathrm{dL}$ ), in which azoospermia results from impaired sperm production. However, rare azoospermic patients with testicular cancer have normal sperm production and incidental obstruction that is unrelated to their malignancy. These patients typically have serum FSH and T within the normal range (FSH $<8$ IU/L and $\mathrm{T}>300 \mathrm{ng} / \mathrm{dL}$ ).

Assessment of serum T and FSH may also enable identification of patients with distinct endocrinologic pathology. Rare patients may be found to have hypogonadotropic hypogonadism (undetectable FSH and low T). Such patients are important to identify, because sperm retrieval is very unlikely to be successful without several months of gonadotropinreplacement therapy. ${ }^{30}$ Other patients may have high serum testosterone levels associated with very low or undetectable FSH. This hormonal profile is consistent with anabolic steroid abuse, which is a common cause of infertility in adolescents and young men. The endocrine evaluation is less helpful in peripubertal adolescents, as normative values for T and FSH have not yet been established in this population.

\section{Options for fertility preservation in adolescents and young adults with testis cancer}

Fertility preservation in adolescents and young men with testis cancer is performed by acquisition and cryostorage of sperm. Acquisition of ejaculated sperm (sperm banking) is simple, inexpensive, and noninvasive, and should be the first strategy attempted in all patients desiring fertility preservation. However, adequate sperm banking is not possible in some cases due to anejaculation, severe deficits in sperm concentration or quality, or azoospermia. Multiple procedures for sperm retrieval are available in such cases and should be selected based upon the clinical scenario (see Figure 2).

\section{Cryopreservation of ejaculated sperm}

Sperm banking by cryopreservation of ejaculated semen is the preferred, simplest, and most cost-effective strategy for fertility preservation, ${ }^{31}$ and has been shown to be feasible even in adolescent populations. ${ }^{32}$ Whenever possible, sperm banking should be attempted prior to induction of antineoplastic treatment, which may cause genetic defects in sperm that could theoretically affect future offspring ${ }^{33}$ and may transiently or permanently impair quantitative sperm production. In patients with newly diagnosed testicular cancer, sperm banking is ideally performed before orchiectomy so as to identify azoospermic patients who may benefit from testicular sperm extraction at the time of radical orchiectomy.

The costs of sperm banking are variable, but typically range from US\$150 to $\$ 400$ for initial sample collection and analysis, and from $\$ 25$ to $\$ 100$ for storage per month. Samples may be produced by masturbation or obtained via specialized collection condoms after 2-3 days of ejaculatory abstinence. In the ideal scenario, multiple semen samples should be collected and analyzed every 48-72 hours to maximize the amount of sperm for cryostorage. However, the abstinence period should not be viewed as mandatory and may be shortened to 24 hours (or less) in patients for whom cancer treatment must be urgently initiated.

\section{Fertility-preservation strategies in patients with ejaculatory dysfunction}

Some testicular cancer patients may be unable to attempt sperm banking. This group includes patients with such advanced disease that their ability to achieve orgasm is limited by physical weakness or pain, patients with primary anorgasmia or severely delayed orgasm, and patients who are religiously or socially prohibited from masturbation. Other patients suffer from neurogenic ejaculatory dysfunction, such as may occur in patients with spinal cord injuries, peripheral neuropathies, and after RPLND for retroperitoneal lymphatic metastases of testicular cancer. The clinical hallmark of these patients is the absence of antegrade ejaculation despite achievement of orgasm.

Options for sperm acquisition in patients unable to produce an antegrade semen sample include penile vibratory stimulation (PVS), electroejaculation, and surgical sperm retrieval. If available, assisted ejaculation procedures may be preferable to sperm retrieval, because higher numbers of sperm will typically be retrieved and many cryobanks lack expertise in handling testicular sperm. Furthermore, if antegrade ejaculation cannot be induced, sperm retrieval can be performed immediately.

PVS is a minimally invasive and effective treatment for anorgasmic testicular cancer patients with spinal cord injuries. The frenular surface of the penis is stimulated with a commercially available vibrator and orgasm is induced. The procedure may be performed by the physician in the office or by the patient at home. PVS is mediated by a spinal reflex arc subject to inhibitory cortical control, and therefore is best suited for patients in whom the reflex arc is intact and cortical inhibition is disrupted, such as men with spinal cord injury above T10. ${ }^{34}$ PVS is not appropriate for men with normal 
Testicular cancer diagnosis

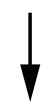

Pre-orchiectomy sperm banking attempt with concurrent semen analysis

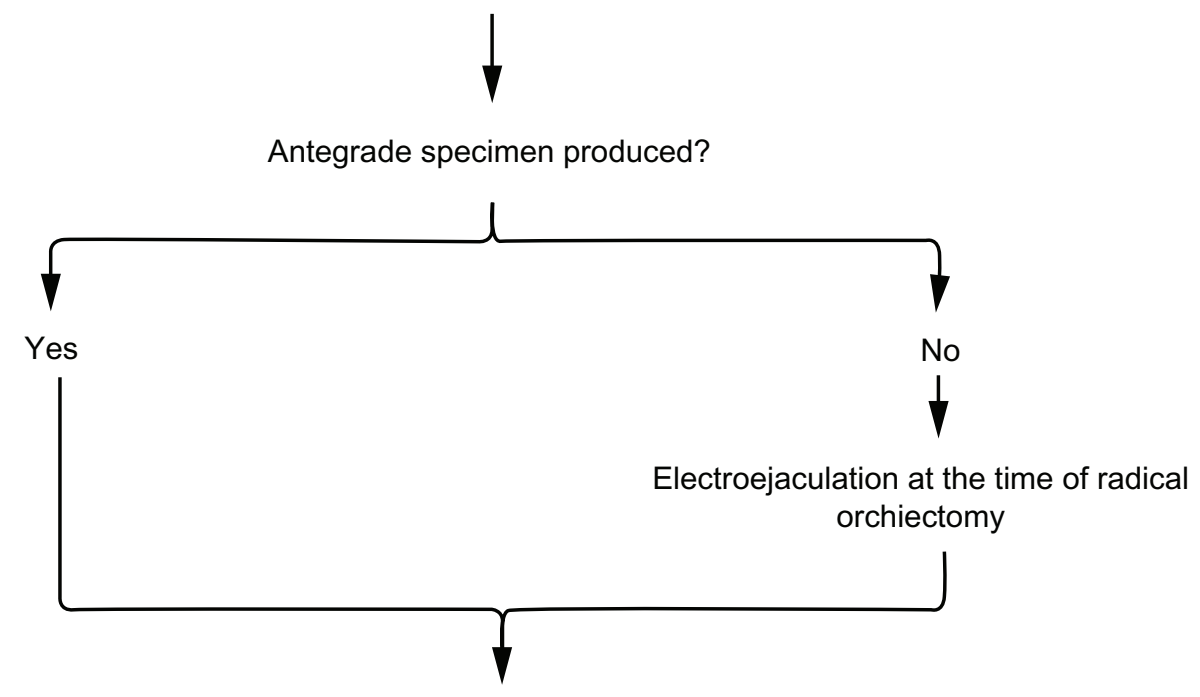

Adequate sperm for cryopreservation?

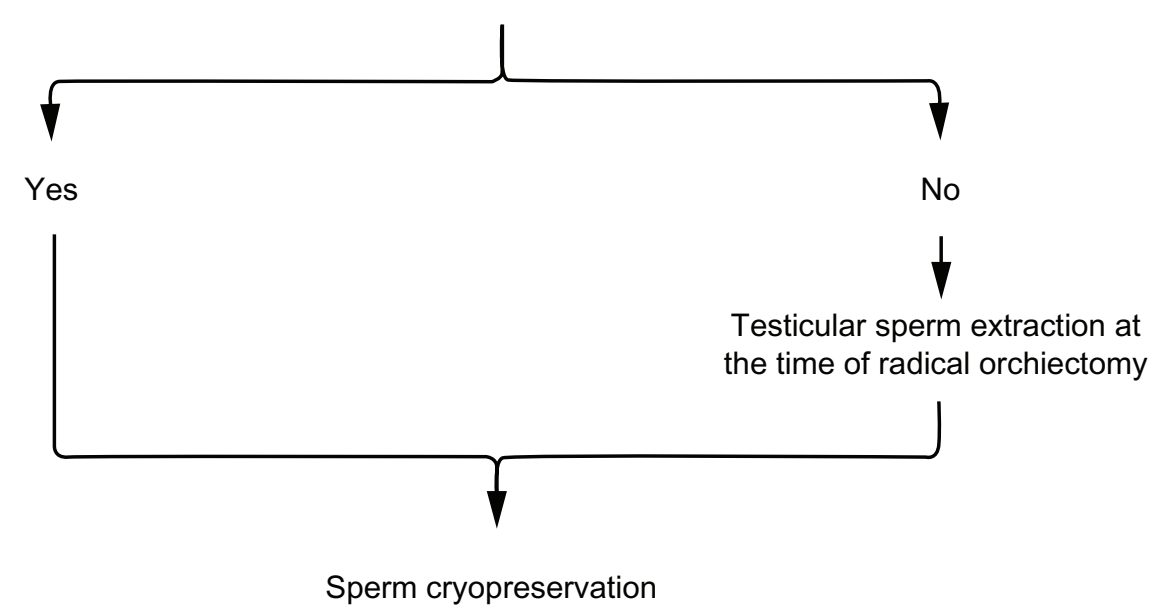

Figure 2 Algorithm for sperm retrieval procedures.

orgasms who suffer from ejaculatory dysfunction (such as after RPLND).

Electroejaculation requires general anesthesia (aside from spinal cord-injured patients) and may be effective for anejaculation of any etiology. The bladder is emptied by urethral catheterization, and a small volume of sperm-transport media is injected into the bladder. The prostate and seminal vesicles are directly electrostimulated with a transrectal probe. Five to 30 volts of energy are delivered cyclically until seminal emission is observed, which may be either pulsatile or occur as a slow drip. Urethral catheterization is used to collect any retrograde component of the ejaculate. Immediate microscopic evaluation of the ejaculated semen is performed in the operating room to assess for the presence of a sufficient number of motile sperm. If there is inadequate sperm recovery, surgical sperm retrieval by testicular sperm extraction may immediately be performed. ${ }^{34}$

\section{Fertility preservation strategies in azoospermic patients}

Azoospermia refers to the state of having zero sperm detectable in the ejaculate. This finding suggests either severe impairment of sperm production (nonobstructive azoospermia) or complete and bilateral obstruction of the male excurrent ductal system (obstructive azoospermia). In some cases, sperm production may improve after removal of 
the cancerous testicle, ${ }^{35}$ or fertility may be restored by future reconstructive microsurgery to bypass obstruction. However, the best hope for biological paternity in the majority of cases is retrieval of testicular sperm for future assisted reproduction via ICSI. It is important to note that testicular sperm cannot be used for simpler methods of assisted reproduction, such as intravaginal insemination or IUI.

Sperm acquisition should be performed using an open surgical approach in testicular cancer patients pursuing fertility preservation. Percutaneous, needle-based methods for testicular sperm extraction are not recommended, as they are less effective ${ }^{36}$ and rely on blind testicular puncture that could put the patient at risk for testicular loss. The optimal procedure is microdissection testicular sperm extraction (TESE) (Figure 3), in which the testicle is opened widely to enable direct inspection of the entire volume of seminiferous tubular tissue using high-power optical magnification provided by an operating microscope. ${ }^{37}$ Larger-diameter tubules are visually identified and extracted with minimal disruption of the testicular blood supply. This approach results in an increased chance of retrieving sperm and a higher number of sperm harvested when compared with traditional, nonmicrosurgical TESE. However, microdissection TESE is not accessible at most centers, and its superiority has not been established in randomized prospective trials. Conventional, nonmagnified TESE is therefore the most commonly utilized procedure for fertility preservation in

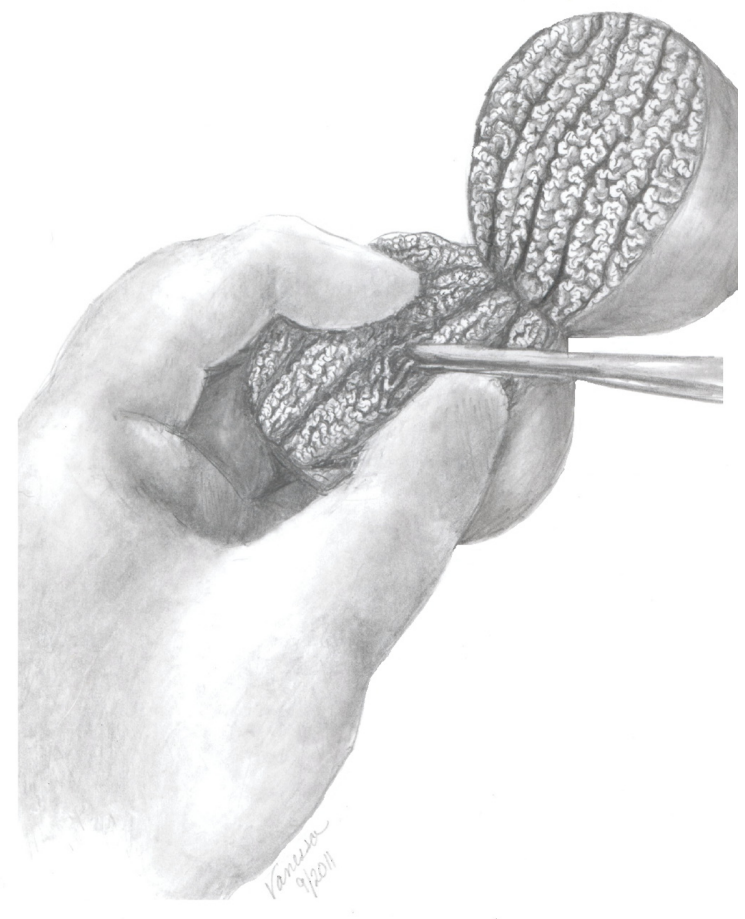

Figure 3 Microdissection testicular sperm extraction. cases of nonobstructive azoospermia associated with cancer. In this approach, the tunica albuginea of the testis is incised in one or multiple areas and seminiferous tubular tissue is sharply excised (Figure 4).

The optimal time for TESE in azoospermic testicular cancer patients is at the time of radical orchiectomy, emphasizing the value of attempting sperm banking prior to orchiectomy. This approach eliminates the need for multiple surgical procedures and minimizes the risk of delaying oncologic therapy for fertility preservation. Sperm may be extracted from the contralateral or tumor-containing testis. Sperm retrieval from the tumor-containing testis is advantageous, because it may be performed ex vivo after completion of the radical orchiectomy and therefore poses no additional risk to the patient. The feasibility of this approach has been demonstrated, and live births of healthy children conceived with sperm extracted from orchiectomy specimens have been reported. . $^{38,39}$

Unfortunately, fertility preservation strategies in azoospermic patients are infrequently utilized due to patient and physician-perceived urgency to initiate treatment and lack of awareness about surgical sperm retrieval. The available data are scarce but encouraging. In the largest published case

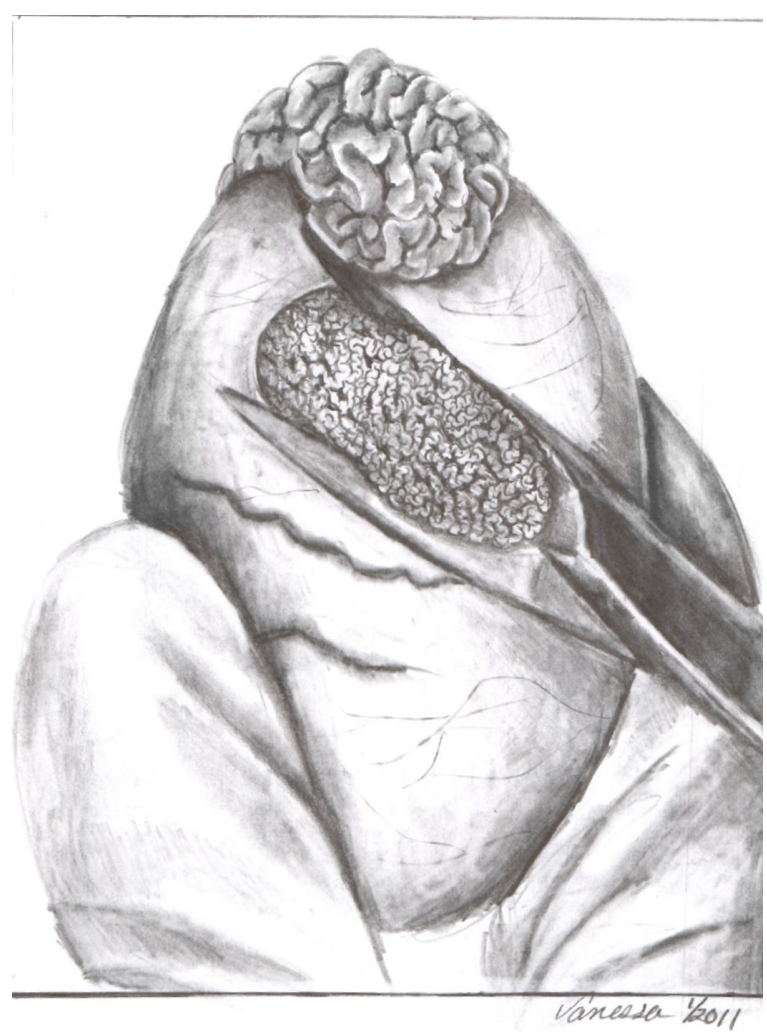

Figure 4 Nonmagnified testicular sperm extraction.

(C) 2012 Springer. Reproduced with permission from Stahl PJ, Stember DS, Mulhall JP. Options for fertility preservation in men and boys with cancer. ${ }^{53}$ 
series, Schrader et al reported successful retrieval of sperm in six of 14 azoospermic men with testicular cancer. ${ }^{40}$

\section{Options for parenthood posttreatment}

As indicated above, barring those males who have had bilateral orchiectomies, retained fertility post-contemporary testicular cancer therapy is possible in many individuals, particularly those with earlier-stage disease. Some patients may initially be azoospermic and then recover adequate spermatogenesis, with the time to recovery being related at least in part to the intensity of therapy. Therefore, it may be reasonable for testicular cancer survivors, even those who banked sperm prior to treatment, to consider conception through intercourse without a prior evaluation. However, unsuccessful attempts at conception through intercourse warrant a semen analysis and consideration of alternative options for attainment of pregnancy.

A series of reports suggest that between $5 \%$ and $25 \%$ of males (with any cancer diagnosis) have used cryopreserved sperm for conception. ${ }^{4,41}$ For those individuals who remain oligospermic or azoospermic posttreatment and who have previously banked sperm, the sperm can be thawed and used in assisted reproduction procedures, including IUI and in vitro fertilization (IVF), if sufficient sperm volume is available, and IVF with ICSI when limited cryopreserved sperm is available. In one study, of the 118 couples that underwent IVF/ICSI utilizing thawed sperm that had been cryopreserved prior to the start of cancer-directed treatment, there was a clinical pregnancy rate of $56.8 \%$ achieved with 96 pregnancies and 126 live children. ${ }^{41}$

Males who did not bank sperm pretreatment and are oligospermic or azoospermic can attempt posttreatment TESE and then IVF utilizing ICSI. Hsiao et al recently reported a sperm-retrieval rate of $37 \%$ using TESE in men previously diagnosed with cancer who were azoospermic after chemotherapy. The subsequent fertilization rate per injected oocyte was $57.1 \%$ and the live birth rate $42 \%$. In a starting sample of 73 men, this translated into a total of 15 deliveries and 20 babies born. Among this sample, those with testicular cancer had the highest sperm-retrieval rates. ${ }^{42}$ In situations where sperm is not available from the testicular cancer survivor, options for parenthood also include the use of donor sperm or adoption.

\section{Psychosocial considerations}

Adolescence and young adulthood are times of critical development. Adolescence is a time of transitions, including changes in physical appearance, cognitive and social development, and emotional growth. Young adulthood is often characterized by being in a committed relationship and starting a family. ${ }^{43}$ These are periods of sexual maturation, and adolescents/young adults are focused on discovering their sexual identity. ${ }^{44}$ The diagnosis of testicular cancer may lead, at least initially, to sexual problems as well as physical and psychological consequences. ${ }^{45}$ Men who are newly diagnosed have reported feeling different from others, believing that they are being perceived as damaged, and not as desirable. ${ }^{46}$ At an age when physical appearance is a major concern, the testicular cancer diagnosis and the side effects of treatment, including the appearance of new scars and the loss of a testicle or body hair, may exacerbate these worries. ${ }^{45}$

Concern about future fertility itself is a source of anxiety for many testicular cancer survivors and causes distress impacting their psychosocial and sexual well-being. ${ }^{47,48}$ Survivors have reported feeling that they received insufficient information regarding fertility-preservation options at diagnosis, and that their fertility was sacrificed unnecessarily. ${ }^{47}$ Banking sperm at the time of diagnosis can serve as a "safety net" and ameliorate some of the worries about being able to have biologic children in the future..$^{49,50}$

\section{Practical considerations: establishing a fertility-preservation program}

The need to preserve fertility comes at a time when patients may be overwhelmed by coping with a new diagnosis and the prospects of upcoming therapy, and their health-care providers may also be focused on coordinating diagnostic and therapeutic surgeries and establishing plans of care. In these instances, attention to the preservation of fertility may be overlooked, especially in scenarios where there are no local sperm banks, the patient is not able to sperm bank, and/or finances are not readily available. Although sperm banking prior to the beginning of treatment has been recommended by both the American Society for Clinical Oncology ${ }^{51}$ and the National Comprehensive Cancer Network, ${ }^{52}$ surveys have suggested that only half of patients are offered the opportunity to bank sperm and half of that group follows through. ${ }^{42}$ Developing routine practices increases the likelihood that patients will be offered fertility-preservation options in the limited amount of time that may be available prior to starting therapy. The type of program that suits an individual practice may vary depending on the size and complexity of the practice, but should at a minimum include information about sperm banks and financial support. 


\section{Identifying a sperm bank}

Maintaining up-to-date information on sperm banks located near a practice or catchment area can facilitate the referral for sperm banking. Relevant information includes days and hours of operation (since some patients may need to bank on the weekend), types of insurance accepted, availability of discounted service rates to cancer patients, familiarity in working with adolescent patients, and logistical ease of the center, including distance from the hospital and accessibility of parking. For larger practices, it can be beneficial to identify people at the sperm banks who may be able to facilitate and expedite the sperm-banking process when time is limited. Patients who cannot go to a sperm bank due to hospitalization may be able to sperm bank while hospitalized and then have the sample transported by a friend or family member to the sperm bank. For those patients who may live too far away or who do not want to delay treatment, mail-in sperm banking kits such as Live:On are available at http:// www.liveonkit.com/order.cfm for centers to have available when needed.

\section{Logistics}

As noted above, options for sites of sperm banking include a sperm bank, an inpatient unit, or a "home" setting. Most sperm banks will have an established process for sperm banking at their site. In all scenarios, however, it is appropriate to explain clearly that masturbation is necessary for the banking process. This is particularly important for younger adolescents, or patients for whom masturbation is traditionally prohibited for religious reasons. For those going to a sperm bank, it is helpful to give the patient the sperm bank's consent ahead of time so they have ample time to read through the document and address any questions or concerns. For the patients who bank while in an inpatient setting, it is imperative to identify a private room, which can be locked from the inside, to provide privacy. Depending on the requirement of the sperm bank, the deposit may need to be collected in a specific container, which will need to be made available ahead of time.

\section{Financial issues}

A range of costs for sperm banking is listed above. These costs may be beyond the means of some patients, especially as it is rare that all of the costs are covered by insurance. Financial assistance programs, such as Livestrongs Sharing Hope program, provide discounts to cancer patients for cryopreservation. In addition, individual sperm banks may provide additional financial assistance to cancer patients, as well as varying payment plans for services.

\section{Patient-navigator role in fertility preservation}

In programs with a high volume of patients at risk of infertility, it is also useful to select a provider at the treating institution to serve as the patient navigator, to screen and consult all patients at risk for fertility problems as a result of cancer diagnosis or treatment. This person should be knowledgeable and comfortable in discussing personal and private issues regarding fertility, including available preservation options, semen obtainment and preservation methods, as well as the processes of using or disposing the banked sperm in the future. They should also provide locations and hours of sperm-bank referral centers, and resources, including printed electronic material or pamphlets that should be available to all patients. An in-service should be provided by the patient navigator to the oncology staff, including physicians and nurses, introducing his/her role, as well as explaining the preservation options and the information that will be explained to patients. In the event that the start of therapy needs to be delayed for sperm banking, it is essential that the other providers understand the rationale for the delay, and the impact the ability to preserve fertility has on the patient's quality of life in the future.

\section{Conclusion}

In the context of contemporary surgical and medical therapy for testicular cancer, many survivors will ultimately regain the ability to sire children. However, a subset of patients will become permanently infertile, and another subset will not regain fertile potential within the timeframe in which they desire biologic children. Although estimates may be made about which patients are likely to fall into the risk categories, accurate assessments cannot be made before the start of therapy, when fertility preservation strategies are most easily employed. For this reason, all patients diagnosed with testicular cancer should routinely be offered the opportunity to sperm bank or be offered alternative methods for sperm collection before therapy is initiated. The probability of successfully offering these services to all patients is maximized by having an established standard operating procedure or program in place.

\section{Disclosure}

The authors have nothing to disclose.

\section{References}

1. Hayes-Lattin B, Nichols CR. Testicular cancer: a prototypic tumor of young adults. Semin Oncol. 2009;36:432-438. 
2. Levine J, Canada A, Stern CJ. Fertility preservation in adolescents and young adults with cancer. J Clin Oncol. 2010;28:4831-4841.

3. Joly F, Héron JF, Kalusinski L, et al. Quality of life in long-term survivors of testicular cancer: a population-based case-control study. J Clin Oncol. 2002;20:73-80.

4. Schover LR. Patient attitudes toward fertility preservation. Pediatr Blood Cancer. 2009;53:281-284.

5. Daneshmand S, Djaladat H, Porter CR, Nichols C. Evaluation and preservation of fertility in patients with testicular cancer. $J$ Adolesc Young Adult Oncol. 2011;1:25-29.

6. Jahnukainen K, Ehmcke J, Hou M, Schlatt S. Testicular function and fertility preservation in male cancer patients. Best Pract Res Clin Endocrinol Metab. 2011;25:287-302.

7. Singh SR, Burnicka-Turek O, Chauhan C, Hou SX. Spermatogonial stem cells, infertility and testicular cancer. J Cell Mol Med. 2011;15: 468-483.

8. Spermon JR, Kiemeney LA, Meuleman EJ, Ramos L, Wetzels AM, Witjes JA. Fertility in men with testicular germ cell tumors. Fertil Steril. 2003;79 Suppl 3:1543-1549.

9. Raman JD, Nobert CF, Goldstein M. Increased incidence of testicular cancer in men presenting with infertility and abnormal semen analysis. J Urol. 2005;174:1819-1822; discussion 1822.

10. Paduch DA. Testicular cancer and male infertility. Curr Opin Urol. 2006; 16:419-427.

11. Huddart RA, Norman A, Moynihan C, et al. Fertility, gonadal and sexual function in survivors of testicular cancer. Br J Cancer. 2005;93: 200-207.

12. Gospodarowicz M. Testicular cancer patients: considerations in longterm follow-up. Hematol Oncol Clin North Am. 2008;22:245-255, vi.

13. Matos E, Skrbinc B, Zakotnik B. Fertility in patients treated for testicular cancer. J Cancer Surviv. 2010;4:274-278.

14. Abouassaly R, Fossa SD, Giwercman A, et al. Sequelae of treatment in long-term survivors of testis cancer. Eur Urol. 2011;60:516-526.

15. Meistrich ML, Wilson G, Brown BW, da Cunha MF, Lipshultz LI. Impact of cyclophosphamide on long-term reduction in sperm count in men treated with combination chemotherapy for Ewing and soft tissue sarcomas. Cancer. 1992;70:2703-2712.

16. Brydøy M, Fosså SD, Klepp O, et al. Paternity and testicular function among testicular cancer survivors treated with two to four cycles of cisplatin-based chemotherapy. Eur Urol. 2010;58:134-140.

17. Brydøy M, Fosså SD, Klepp O, et al. Paternity following treatment for testicular cancer. J Natl Cancer Inst. 2005;97:1580-1588.

18. Sklar C. Reproductive physiology and treatment-related loss of sex hormone production. Med Pediatr Oncol. 1999;33:2-8.

19. Centola GM, Keller JW, Henzler M, Rubin P. Effect of low-dose testicular irradiation on sperm count and fertility in patients with testicular seminoma. J Androl. 1994;15:608-613.

20. Hahn EW, Feingold SM, Simpson L, Batata M. Recovery from aspermia induced by low-dose radiation in seminoma patients. Cancer. 1982;50: 337-340.

21. Shalet SM, Tsatsoulis A, Whitehead E, Read G. Vulnerability of the human Leydig cell to radiation damage is dependent upon age. J Endocrinol. 1989;120:161-165.

22. Ragni G, Somigliana E, Restelli L, Salvi R, Arnoldi M, Paffoni A. Sperm banking and rate of assisted reproduction treatment: insights from a 15-year cryopreservation program for male cancer patients. Cancer. 2003;97:1624-1629.

23. Williams DH 4th, Karpman E, Sander JC, Spiess PE, Pisters LL, Lipshultz LI. Pretreatment semen parameters in men with cancer. J Urol. 2009;181:736-740.

24. Cooper TG. WHO Laboratory Manual for the Examination and Processing of Human Semen, 5th ed. Geneva: World Health Organization; 2010.

25. Kuczyński W, Dhont M, Grygoruk C, Grochowski D, Wołczyński S, Szamatowicz M. The outcome of intracytoplasmic injection of fresh and cryopreserved ejaculated spermatozoa - a prospective randomized study. Hum Reprod. 2001;16:2109-2113.
26. Stahl PJ, Stember DS, Goldstein M. Contemporary management of male infertility. Аnпu Rev Med. 2012;63:525-540.

27. Wood HM, Elder JS. Cryptorchidism and testicular cancer: separating fact from fiction. J Urol. 2009;181:452-461.

28. Sheynkin YR, Hendin BN, Schlegel PN, Goldstein M. Microsurgical repair of iatrogenic injury to the vas deferens. J Urol. 1998;159: 139-141.

29. Hagenas I, Jorgensen N, Rechnitzer C, et al. Clinical and biochemical correlates of successful semen collection for cryopreservation from 12-18-year-old patients: a single-center study of 86 adolescents. Hum Reprod. 2010;25:2031-2038.

30. Sokol RZ. Endocrinology of male infertility: evaluation and treatment. Semin Reprod Med. 2009;27:149-158.

31. Jeruss JS, WoodruffTK. Preservation of fertility in patients with cancer. N Engl J Med. 2009;360:902-911.

32. Keene DJ, Sajjad Y, Makin G, Cervellione RM. Sperm banking in the United Kingdom is feasible in patients 13 years old or older with cancer. J Urol. 2012;188:594-597.

33. Robbins WA, Meistrich ML, Moore D, et al. Chemotherapy induces transient sex chromosomal and autosomal aneuploidy in human sperm. Nat Genet. 1997;16:74-78.

34. Ohl DA, Quallich SA, Sønksen J, Brackett NL, Lynne CM. Anejaculation: an electrifying approach. Semin Reprod Med. 2009;27:179-185.

35. Lampe H, Horwich A, Norman A, Nicholls J, Dearnaley DP. Fertility after chemotherapy for testicular germ cell cancers. J Clin Oncol. 1997; 15:239-245.

36. Friedler S, Raziel A, Strassburger D, Soffer Y, Komarovsky D, Ron-El R. Testicular sperm retrieval by percutaneous fine needle sperm aspiration compared with testicular sperm extraction by open biopsy in men with non-obstructive azoospermia. Hum Reprod. 1997;12: 1488-1493.

37. Schlegel PN. Testicular sperm extraction: microdissection improves sperm yield with minimal tissue excision. Hum Reprod. 1999;14: 131-135.

38. Baniel J, Sella A. Sperm extraction at orchiectomy for testis cancer. Fertil Steril. 2001;75:260-262.

39. Descombe L, Chauleur C, Gentil-Perret A, Aknin-Seifer I, Tostain J, Lévy R. Testicular sperm extraction in a single cancerous testicle in patients with azoospermia: a case report. Fertil Steril. 2008;90:443. e1-e4.

40. Schrader M, Müller M, Sofikitis N, Straub B, Krause H, Miller K. "Onco-tese": testicular sperm extraction in azoospermic cancer patients before chemotherapy - new guidelines? Urology. 2003;61: 421-425.

41. Hourvitz A, Goldschlag DE, Davis OK, Gosden LV, Palermo GD, Rosenwaks Z. Intracytoplasmic sperm injection (ICSI) using cryopreserved sperm from men with malignant neoplasm yields high pregnancy rates. Fertil Steril. 2008;90:557-563.

42. Hsiao W, Stahl PJ, Osterberg EC, et al. Successful treatment of postchemotherapy azoospermia with microsurgical testicular sperm extraction: the Weill Cornell experience. J Clin Oncol. 2011;29: 1607-1611.

43. Tuinman MA, Hoekstra HJ, Fleer J, Sleijfer DT, Hoekstra-Weebers JE. Self-esteem, social support, and mental health in survivors of testicular cancer: a comparison based on relationship status. Urol Oncol. 2006;24: 279-286.

44. Collins WA, Stroufe LA. Capacity for intimate relationship: a developmental construction. In Furman W, editor. The Development of Romantic Relationships in Adolescence. New York: Cambridge University Press; 1999:125-147.

45. Tuinman MA, Hoekstra HJ, Vidrine DJ, et al. Sexual function, depressive symptoms and marital status in nonseminoma testicular cancer patients: a longitudinal study. Psychooncology. 2010;19:238-247.

46. Carpentier MY, Fortenberry JD, Ott MA, Brames MJ, Einhorn LH. Perceptions of masculinity and self-image in adolescent and young adult testicular cancer survivors: implications for romantic and sexual relationships. Psychooncology. 2011;20:738-745. 
47. Schover LR, Brey K, Lichtin A, Lipshultz LI, Jeha S. Knowledge and experience regarding cancer, infertility, and sperm banking in younger male survivors. J Clin Oncol. 2002;20:1880-1889.

48. Zebrack BJ, Casillas J, Nohr L, Adams H, Zeltzer LK. Fertility issues for young adult survivors of childhood cancer. Psychooncology. 2004;13: 689-699.

49. Saito K, Suzuki K, Iwasaki A, Yumura Y, Kubota Y. Sperm cryopreservation before cancer chemotherapy helps in the emotional battle against cancer. Cancer. 2005;104:521-524.

50. Ginsberg JP, Ogle SK, Tuchman LK, et al. Sperm banking for adolescent and young adult cancer patients: sperm quality, patient, and parent perspectives. Pediatr Blood Cancer. 2008;50:594-598.
51. Lee SJ, Schover LR, Partridge AH, et al. American Society of Clinical Oncology recommendations on fertility preservation in cancer patients. J Clin Oncol. 2006;24:2917-2931.

52. Coccia PF, Altman J, Bhatia S, et al. Adolescent and young adult oncology. J Natl Compr Canc Netw. 2012;10:1112-1150.

53. Stahl PJ, Stember DS, Mulhall JP. Options for fertility preservation in men and boys with cancer. Springer; 2012. Available at http://chp. broadcastmed.net/files/dmfile/FertPreservation2.pdf.

\section{Publish your work in this journal}

Clinical Oncology in Adolescents and Young Adults is an international, peer-reviewed, open access journal publishing original research, reports, editorials, reviews and commentaries on all aspects of epidemiology, diagnosis and treatment of cancers in adolescents and young adults. The manuscript management system is completely online and includes a very quick and fair peer-review system. Visit http://www.dovepress.com/testimonials.php to read real quotes from published authors.

\footnotetext{
Submit your manuscript here: http://www.dovepress.com/clinical-oncology-in-adolescents-and-young-adults-journal
} 